\title{
EL DESEMPEÑO DEL PROFESOR Y LA RELACIÓN DE AFECTIVIDAD Y APRENDIZAJE DESDE LA PERSPECTIVA DE PIAGET Y WALLON
}

\section{ARTÍCULO DE REVISIÓN}

DIAS, Adailton di Lauro ${ }^{1}$

DIAS, Adailton di Lauro. El desempeño del maestro y la relación de afectividad y aprendizaje desde la perspectiva de Piaget y Wallon. Revista Científica Multidisciplinar Núcleo do Conhecimento. Ano 04, Ed. 07, Vol. 09, págs. 64-71. Julio de 2019. ISSN: 2448-0959

\section{RESUMEN}

El estudio aborda el tema de la relación de la afectividad y el aprendizaje. Cita la importancia del desempeño del maestro en este contexto. Aborda que la afectividad, la cognición y la motivación colaboran en el desarrollo del aprendizaje del alumno a través de la mediación del profesorado en la escuela diaria. Se basa en teóricos como Piaget y Wallon. Tiene como objetivo general entender la relación entre aprendizaje y afectividad desde la perspectiva de Piaget y Wallon. Trae como un problema de investigación: ¿Cómo puede la afectividad, la cognición y la motivación colaborar en el desarrollo del aprendizaje del alumno con la mediación del profesor en el aula? A partir de los estudios realizados, se considera que, desde la perspectiva de Piaget, en su teoría piagetiana, la afectividad, la cognición y la motivación son las condiciones necesarias en la constitución de la inteligencia y el aprendizaje significativo. En opinión de Valonía, en su teoría llamada Valonia, para el aprendizaje, es necesario integrar entre conjuntos funcionales (afectividad, cognición, motricidad y persona). Por lo tanto, es necesario enfatizar la importancia de las relaciones establecidas con los aspectos emocionales de los estudiantes en el proceso de enseñanza y aprendizaje. Y que las

\footnotetext{
${ }^{1}$ Mestrando em Ciências da Educação (Grendal University). Especialista em Língua Inglesa (FIJ). Graduado em Letras (UNEB). Graduado em Português e Inglês pela Universidade Metropolitana de Santos - SP. Professor EBTT Port/Ing - IFRR.
} 
relaciones que implican afectividad pueden afectarse mutuamente en tu comportamiento, ayudándote a adquirir conocimiento más fácilmente, haciéndolo más inteligente, impulsándolo y motivándolo a acciones que involucran el aprendizaje Significativo. Así, la afectividad, la cognición y la motivación ayudan en el desarrollo del aprendizaje de los estudiantes en la vida escolar diaria.

Palabras clave: afecto, aprendizaje, profesor, Piaget, Valon.

\section{INTRODUCCIÓN}

El estudio analiza la relación entre aprendizaje y afectividad, destacando en este proceso, la importancia del desempeño del maestro en el aula, demostrando que su estado emocional, así como el de los alumnos se refleja positiva o negativamente en el aprendizaje. También trae el tema de la afectividad, la cognición y la motivación como factores primarios para el desarrollo y aprendizaje del estudiante, desde la perspectiva de Piaget y Wallon.

El problema del estudio busca cuestionar: ¿Cómo puede la afectividad, la cognición y la motivación colaborar en el desarrollo del aprendizaje del alumno con la mediación del profesor en el aula? Para lograr el objetivo general que se configura en la comprensión de la relación entre aprendizaje y afectividad desde la perspectiva de Piaget y Valony también para responder al problema de este estudio, se utilizó la investigación bibliográfica. Las reflexiones sobre la relación entre el aprendizaje y el desarrollo de los estudiantes motivaron la construcción de este trabajo.

\section{EL AFFECTION, LA COGNITION Y LA MOTIVACION COMO FACTORES PRIMARIOS PARA EL DESARROLLO Y APRENDIZAJE DEL ESTUDIANTE}

La afectividad, la cognición y la motivación son factores esenciales para el desarrollo y el aprendizaje de los estudiantes en la escuela diaria, incluyendo el desarrollo de inteligencia, ya que, 
(...) sin afecto, no habría interés, no habría necesidad, no hay motivación; y en consecuencia, las preguntas o problemas nunca se plantearían y no habría inteligencia. La afectividad es una condición necesaria en la constitución de la inteligencia (PIAGET, 1992, p. 32).

La educación occidental tiene como patrimonio cultural, la desconexión del hombre de sus emociones, sus pensamientos, su propia realidad, dando lugar a la formación de individuos débiles de espíritu, energía e ignorancia emocional. Esta es, por lo tanto, una visión fragmentada del hombre, que lamentablemente contribuye a una ruptura en las relaciones humanas. Sin embargo, estos dualismos tradicionales del pensamiento occidental presentes en la historia de la psicología tradicional fueron confrontados y cuestionados por teorías innovadoras con el objetivo de integrar, dialécticamente, la cognición y la afectividad, la razón y la emoción.

Sin embargo, la afectividad es indispensable para el desarrollo intelectual de los niños y adolescentes, que los impulsa a actuar en el objeto del conocimiento. "Pero la afectividad no es nada sin inteligencia, que les proporciona los medios y aclara los fines" (PIAGET, 1992, p.70). En este sentido, la inteligencia actúa en afectiva, considerablemente a lo largo del proceso de existencia humana, en el que la organización diaria proporciona a la construcción activa, elementos favorables a la formación de la personalidad.

Así, es posible entender las relaciones entre el afecto y la inteligencia, esto corresponde a las emociones que se vuelven al mundo externo, orientadas al mundo de los objetos de estudio y observación. Lo que se llama inteligencia es la atención que el niño y el adolescente comienzan a dirigir a los componentes del mundo que los rodea, con el fin de tratar de entender cómo funcionan las cosas para adaptarse a ellos.

Es la interrelación recíproca de estos dos casos, afectivo y racional, que se produce la formación y el desarrollo de la personalidad, que aquí se entiende como el producto de la relación que se construye entre el sujeto y el entorno sociocultural, dando lugar a un ser único, individual. 
Piaget (1971) advirtió que, a pesar de que es diferente en su naturaleza, la afectividad y la cognición son inseparables, porque en todas las acciones simbólicas y sin sentido parecen indisociadas. Documentó el comportamiento efectivo de la acción y el pensamiento como un aspecto cognitivo, a través de estructuras mentales, y un aspecto afectivo, a través de una energía, que es la afectividad.

Vygotsky (2002) entiende al hombre como un ser que tiene la capacidad de pensar, razonar, deducir y abstracto, pero también como alguien dotado de sentimientos, emociones, deseos, imaginación y sensibilización al mundo exterior. Por lo tanto, no se puede analizar la sensación y la razón por separado. Más bien, un análisis en su totalidad tiene sentido, porque indica subvenciones favorables a posibles soluciones sobre los problemas existentes. Porque "demuestra la existencia de un sistema dinámico de significados en el que se unen afectivos e intelectuales" (VYGOTSKY, 2002, p. 7). Sin embargo, es indispensable reforzar el significado de las relaciones que los educadores establecen con los aspectos emocionales de los estudiantes en el proceso de enseñanza y aprendizaje.

En cuanto a las emociones, Wallon (apud GALV-O, 1995) dice que ha sido desde el comienzo de la vida; lo defiende, como el primer y más fuerte vínculo entre los individuos. Al principio de su existencia, el niño gradualmente se convierte en un ser sociocognitivo al construir gradualmente, una visión única y particular de su existencia.

Wallon (apud GALV-O, 1995) también afirma que la afectividad aparece cuando surgen elementos simbólicos, es decir, se manifiesta desde el contacto con el otro, deja claro que la afectividad implica un conjunto de manifestaciones que abarca sentimientos y emociones.

Dentro de esta consideración Galvo aborda en el texto "expresividad y emociones, de acuerdo con la perspectiva de Valonia" que:

Al principio, a través de sus gestos impulsivos, contorsiones o espasmos corporales, así como las expresiones emocionales más primitivas, como llorar o sonreír, el bebé humano moviliza a la gente de su entorno en una 
especie de contagio afectivo. El adulto interpreta, en consecuencia, sus valores, deseos y sus expectativas, el significado de las expresiones emocionales del bebé, siendo llevado a actuar de acuerdo con sus parámetros culturales, deseos y sus creencias individuales, envueltos en el clima de contagio propio de estos (GALV-O, 1995, p. 74).

Sin embargo, de esta manera que el bebé humano tiene que expresarse son manifestaciones de pura emoción y provocar reacciones afectivas que los inducen a satisfacer las necesidades del niño. En este sentido, la afectividad es el comportamiento capaz de afectar al otro, de modo que lo lleva a la acción. La primera forma de afecto reside en las emociones, cuyo papel es unir a los individuos entre sí mediante reacciones orgánicas e íntimas de una manera global e indiferenciada.

Es a través de esta unión que los estudiantes cuando llegan a la escuela se relacionan entre sí, y ganan avances significativos en la esfera cognitiva. Con esto, los lazos afectivos se expanden y el maestro juega un papel importante en el desarrollo de los estudiantes en el contexto escolar.

Para adherirse al comportamiento motivacional a los aspectos afectivos y cognitivos, vale la pena mencionar que:

El desarrollo de la inteligencia, sin duda, permite que la motivación se despierte por un número cada vez mayor de objetos o situaciones. Sin embargo, a lo largo de este desarrollo, el principio básico sigue siendo el mismo: La afectividad es el resorte impulsor de las acciones, y la razón está a su servicio (LA TAILLE, 1992, p. 65).

La motivación, a su vez, es una parte integral del proceso de enseñanza y aprendizaje, es uno de los elementos considerables y esenciales para aprender o lograr algo. Todas las acciones son movidas por una fuerza motivacional. "La motivación es la fuerza motriz de la conducta. Es la condición interna que activa al individuo y le predispone a dar ciertas respuestas" (CORIA-SABINI, 2004, p. 83). 
Sin embargo, para aprender o enseñar, es necesario que se produzca una fuerza motriz motivacional. La ausencia de este día a día en la práctica educativa tanto en el estudiante como en la dirección y en la facultad perjudicará el desarrollo cognitivo de los estudiantes.

\subsection{AFECTABILIDAD Y SU RELACION CON EL APRENDIZAJE: EL RENDIMIENTO DEL PROFESOR EN EL CLASE}

Entender la importancia de la actuación del maestro en el aula, se percibe que su estado emocional, así como el de los estudiantes se refleja en el aprendizaje. El maestro al mostrar afecto en sus acciones, motiva al alumno a querer participar activamente en sus clases, percibiendo al maestro como un amigo siempre dispuesto a ayudarlo y enseñarle.

Para Oliveira y Chadwick (2001, p. 52),

El estado emocional y afectivo de los estudiantes influye en su aprendizaje. La motivación es como un desencadenante que impulsa el aprendizaje y establece las condiciones en las que se produce. Las emociones juegan un papel importante en el aprendizaje en cualquier etapa de la vida. Pero son particularmente importantes en los estudiantes más jóvenes, ya que la situación escolar es bastante artificial en relación con sus vidas y no es muy compatible con sus preferencias.

La práctica de un maestro afectivo será respetar el ritmo de aprendizaje de cada alumno, porque tiene la sensibilidad de percibir cuándo o no proceder con el contenido. Un estudiante puede necesitar más tiempo para aprender una cierta disciplina que su colega, pero también puede ser más rápido en una disciplina que tiene más afinidad.

Como afirma Mendes (2017), es necesario reflexionar sobre la práctica pedagógica y la formación del profesorado. Afirma que la educación continua reflexiona sobre el papel del educador como profesional, sin embargo esta formación se refleja, 
especialmente, en este profesional como persona, porque las personas terminan siendo influenciadas por otras personas y por lo que aprenden en decisiones o decisiones y lo que se transmitirá en la escuela diaria. La teoría elegida por este autor es valoniana, cuyo punto principal es integrar conjuntos funcionales - afectividad, cognición, motricidad y la persona y también el organismo medio.

Por lo tanto, para Valón (2007), esto proporciona la comprensión de la persona en su totalidad, entendiéndola no sólo desde la perspectiva de uno de los sets, sino a través de la integración continua entre ellos. El estudio de Henri Wallon (1999) también hace hincapié en el entorno en el que se inserta la persona, ya que tanto el individuo como la persona sufren influencia entre sí. Desde esta perspectiva, Wallon considera que este elemento es igualmente importante en su proceso de desarrollo.

Para que el profesor haga un buen trabajo, con el objetivo de llegar al mayor número posible de estudiantes, necesita utilizar diversas técnicas de enseñanza, adoptando no sólo obras individuales, sino también en parejas, grupales, etc. Además, también es importante que los maestros tengan una buena relación con otros maestros de la escuela, para intercambiar información sobre los estudiantes dentro de cada área del conocimiento.

En este contexto el profesor se encarga de promover medios que aseguren la motivación, en la que conducirá al aprendizaje. Con respecto a este Coria-Sabini aborda:

La motivación tiene un aspecto cíclico. En primer lugar, existe una necesidad que se manifiesta a través de la estimulación interna específica. En segundo lugar, hay acciones encaminadas a lograr un objetivo. Una vez alcanzado este objetivo, se siguen los alivios y la disminución de la tensión. Este alivio es temporal y después de algún tiempo el ciclo se reanuda. Un bebé que llora se calma después de alimentarse. Sin embargo, horas más tarde el hambre reaparece y debe ser reinsatisfecho (CORIA-SABINI, 2004, p. 84). 
De hecho, esto es lo que debe suceder en el aula, el maestro debe tener estrategias que motiven al estudiante a actuar sobre el objeto del conocimiento; cuando el estudiante se las arregla para sacar sus conclusiones se sentirá aliviado. De aquí es el que entra de nuevo el profesor trayendo nuevas problemáticas y el estudiante irá una vez más en busca del nuevo conocimiento. Cada vez que el estudiante alcanza la meta propuesta, le confunde al maestro proponer otros desafíos para que el estudiante continúe desarrollando su potencial.

En cuanto a los medios o metodologías que el profesor utiliza para motivar a sus alumnos no acuden al caso, cada uno trabaja de manera diferenciada, lo que importa es trabajar de manera que el alumno perciba el aprendizaje como un logro personal. Como, por ejemplo, un estudiante realiza una determinada actividad que consideró incapaz de cumplirla, después de que el maestro le ofreció un brindis desafiándolo a ejecutarlo. Con esto, el estudiante puede sacar sus conclusiones de que es capaz de realizar la actividad sin necesidad de premios, sino como un logro personal.

Sin embargo, los enfoques de los autores citados, como Piaget, Vygotsky y Wallon demuestran cómo la emoción junto con la razón juega un papel esencial en el aprendizaje de los estudiantes. Según ellos, el trabajo del maestro no puede limitarse a la mera memorización, sino a sentir y emocionar con ellos. Desafortunadamente, esta comprensión de la relevancia y el papel adoptado por las emociones en el proceso de enseñanza y aprendizaje es rara entre los maestros, ya que la información que guía esta idea no se transmite, y todavía teniendo en cuenta que las teorías educativas, como el tecnicismo, que sustentan la práctica pedagógica, no dan el debido valor a este aspecto emocional del desarrollo humano.

Saltini cita nuevos paradigmas en la educación, entre ellos, vale la pena mencionar lo que dice:

Habrá un espacio y tiempo dentro de la escuela en el que pueda destacar a mis compañeros de clase, el mundo en el que vivo, mis ideas, fantasías, mis descubrimientos, invenciones, deseos y sueños. Estos, emergiendo de nuestro núcleo, se unen y tienen sentido en los objetos 
del mundo exterior, es decir, cada uno dentro de sí mismo construye su propio vínculo, creando sus símbolos y valores, así como su significado especial, y es a través de estos mismos significados que el sujeto crea vínculos entre el mundo interior. A partir de esto y con esto debemos respetar y crear una metodología pedagógica que conduzca al tema a su pleno desarrollo (SALTINI, 2008, p. 31).

En este sentido, se espera que la escuela se convierta en un ambiente de investigación, en el que el tema de este proceso pueda expresar sus formas de pensamiento, sus dudas y descubrimientos, para que mejore su capacidad comunicativa y se amplíe, significativamente, su inserción en el espacio que desarrolla el potencial de los estudiantes no sólo en el campo de la racionalidad, sino también en el campo de las emociones.

En resumen, Piaget, además de priorizar la importancia de la maduración biológica para la adquisición del aprendizaje, también consideró la importancia de la interacción para la construcción del conocimiento. Vigotsky tenía toda su teoría apoyada en la interacción entre el individuo y el medio. Para que el intercambio entre compañeros fuera más eficaz, destacó el papel del lenguaje para la expresión del pensamiento. Centrándose en la relación entre los dominios afectivos y cognitivos, Wallon creó la teoría del desarrollo de la personalidad, destacando que esto se forma entre la afectividad y la cognición. Además, señaló que la afectividad es una de las fases más antiguas en desarrollo.

\section{CONCLUSIÓN}

El estudio hace hincapié en la necesidad del maestro de buscar en el aula, la integración entre

afectivas y cognitivas en el proceso de enseñanza y aprendizaje, ya que la afectividad y la cognición están interconectadas. En este contexto, el desarrollo de la conectividad precisa de las elaboraciones llevadas a cabo en el plan de inteligencia, y también, el desarrollo de la inteligencia necesita las construcciones afectivas. 
La afectividad sigue siendo un campo amplio para la investigación, se notó a través de este estudio que es extremadamente importante para el proceso educativo y especialmente para establecer una buena relación entre el profesor y el estudiante.

Desafortunadamente, durante su desarrollo, los niños a menudo crecen en un ambiente donde las personas no se respetan mutuamente, viviendo en hostilidad, donde la afectividad está ausente. La tendencia es que estos niños se vuelvan agresivos tanto en sus familias como en la escuela. Desde el momento en que los niños se vuelven agresivos, surgirán dificultades en las relaciones sociales. Por lo tanto, es necesario que el maestro sepa cómo tratar con sus alumnos demostrando afecto en sus acciones.

Sin embargo, el educador debe ser consciente de que no es suficiente ser sólo afectivo para que el estudiante aprenda. Para ello, el profesor debe, en primer lugar, ser un investigador, preocupado por su práctica pedagógica, comprometido con su función social y ética en sus acciones.

La afectividad es sólo un camino para mejorar la relación profesor-alumno, lo que contribuirá al éxito escolar de ambos. Es a través del afecto en el momento del diálogo entre todos los miembros de la comunidad escolar que las dudas pueden ser subsanadas, la indisciplina puede ser eludeda y el deseo del estudiante de aprender será alto y el aprendizaje logrado.

\section{REFERENCIAS}

CORIA-SABINI, Maria Aparecida. Psicologia do Desenvolvimento. 2. ed. São Paulo: Ática. 2004.

GALVÃO, Izabel. Henri Wallon: Uma concepção dialética do desenvolvimento infantil. Petrópolis, RJ: Vozes, 1995.

LA TAILLE, Yves de; OLIVEIRA, Marta Kohl de; DANTAS, Heloysa. Piaget, Vygotsky e Wallon: teorias psicogenéticas em discussão. 13. ed. São Paulo: Summus, 1992. 
MENDES, Daniela Barros. Memórias afetivas: A constituição do professor na perspectiva de Henri Wallon. Sao Paulo: Loyola, 2017.

PIAGET, Jean. A equilibração das estruturas cognitivas. Problema central do desenvolvimento. Trad. Álvaro Cabral. Rio de Janeiro: Zahar, 1976.

. Desenvolvimento e aprendizagem. In: BRINGUIER, J.; PIANCELLA, J.R NESS. J.S.V., Org. In: Studying teaching. 2. ed. Prentice-Hall, 1971.

SALTINI, Cláudio J. P. Afetividade e Inteligência. 5. ed. - Rio de Janeiro: Wak Editora. 2008.

VYGOTSKY, Lev Semenovich. A formação social da mente: o desenvolvimento dos processos psicológicos superiores. 6. ed. São Paulo: Martins Fontes, 2002.

WALLON, Henri. A evolução psicológica da criança. Lisboa: Edições 70, 1999. . Afetividade e aprendizagem - Contribuições de Henry Wallon. São Paulo: Edições Loyola, 2007.

Enviado: Junio de 2019.

Aprobado: Julio de 2019. 